\title{
An Adaptive Gain Tuner Based Fore-ground Calibration Algorithm of a Pipeline ADC
}

\author{
Shatadal Chatterjee, IIITG and Sounak Roy, IIITG
}

\begin{abstract}
This brief proposes a low power mixed-signal foreground calibration algorithm of a pipeline ADC, using a digitally controlled re-configurable switched capacitor multiplying DAC (MDAC) gain controller. The proposed calibration technique forces the front-end stage MDAC gain towards its ideal value to achieve the ideal ADC output linearity. In this paper, a feedback mechanism has been employed to nullify the effect of change in MDAC gain from its ideal value by sensing a digital backend unit response. The proposed technique has been verified in $0.18 \mu \mathrm{m}$ CMOS process using the cadence virtuoso tool by simulating an 11- bit pipeline ADC which contains a 1.5-bit nonideal pipeline stage followed by a 10-bit linear back-end ADC (BE-ADC). Simulation results demonstrate the recovery of the lost linearity of a 11 bit pipeline ADC post calibration.

Index Terms-Pipelined-ADC, Foreground Calibration, MDAC Gain, Adaptive Gain Tuning, Mixed-Signal.
\end{abstract}

\section{INTRODUCTION}

$\mathbf{P}$ IPELINED ADCs [1]-[3] are very useful for wireless communication applications because of high speed, high resolution, and low power consumption at the same time. As the process technology nodes are shrinking every year, reduction of power consumption becomes one of the critical design concerns [4]. To reduce the power budget of a high resolution Pipeline ADCs, reducing the intrinsic gains of building blocks such as amplifiers are essential, resulting in non-linearity at the ADC output [5]. In order to counter the effect of non-linearity at the ADC outputs, calibration has become a popular choice [4], [6]-[15] to meet the growing specification demand. In this paper a low power mixed signal foreground calibration technique has been proposed based on a digitally assisted dynamic switched capacitor gain tuner. Unlike radix extraction based foreground calibration technique ([8], [12]-[15]), the proposed method forces the MDAC gain value of a pipeline stage to its ideal value by deliberately skewing the ideal passive component ratio and proper choice of amplifier open loop gain [11]. The advantage of using the proposed calibration algorithm over the radix extraction based calibration algorithm is two fold. Firstly, in case of the radix extraction based calibration algorithm, the ADC output bit streams are not calibrated; instead, it calibrates an ADC output code which is of the form $D_{\text {out }}=r a_{1} D_{1}+D_{B E}$, by accurately extracting the value of $r a_{1}$. The proposed calibration algorithm, on the other hand, calibrates the ADC output bit-stream itself. In doing so, the proposed method calibrates the relevant stage gain to its ideal value, rather than extracting its actual value. Secondly, typical radix extraction based algorithms often requires complex back end post processing to generate the correct radices ([6], [8] and, [12]-[15]), e.g., an iteration and update algorithm. In the proposed method, a fairly simple feedback mechanism does the job of calibrating the ADC output, without the need of an iteration and update method. Further, this method calibrates a pipeline stage within a very few clock cycles, in the order of 10 . Summary of few existing works are given in Table I.

TABLE I: LITERATURE SURVEY

\begin{tabular}{|c|c|c|c|c|}
\hline Ref. & $\begin{array}{c}\text { Gain } \\
\text { Error } \\
\text { Calibration }\end{array}$ & $\begin{array}{c}\text { Capacitor } \\
\text { Mismatch } \\
\text { Calibration }\end{array}$ & $\begin{array}{c}\text { Radix } \\
\text { Extraction } \\
\text { based }\end{array}$ & $\begin{array}{c}\text { Update } \\
\text { and Iteration } \\
\text { Based }\end{array}$ \\
\hline$[6]$ & YES & YES & YES & YES \\
\hline$[12]$ & YES & YES & YES & NO \\
\hline$[15]$ & YES & YES & YES & NO \\
\hline$[$ Pro. $]$ & YES & YES & NO & NO \\
\hline
\end{tabular}

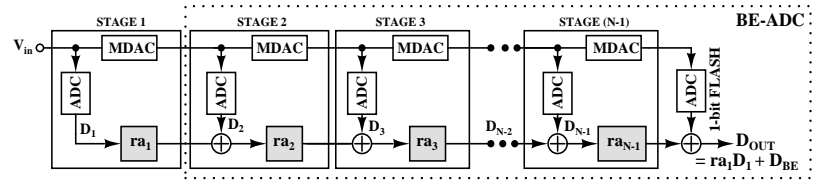

Fig. 1: Block Diagram of a Pipeline ADC

In this paper, an 11 bit pipeline ADC, as shown in Fig. 1, has been considered for illustration. In this ADC, 1st stage is under calibration. Rest of the stages, forming a 10 bit linear back-end ADC (BE-ADC), helps in the calibration process. As shown in [8], [9], [10] and [12]-[14], BE-ADC outputs are used as data for the post processing in order to carry out objectives such as extraction of radices of the target stages. It may be noted here that using an $\mathrm{N}$ bit BE-ADC for post processing is a fairly standard practice for algorithms using radix extraction. The proposed method, although does not use radix extraction, uses the linearity of the BE-ADC to accurately calibrate a pipeline stage. The paper is organized as follows: Section II explains the proposed calibration mechanism. Section III discusses the circuit implementation of the calibration block. The Simulation results are given in section IV followed by conclusions in Section V.

\section{Proposed Calibration Technique}

Fig. 2 shows the block diagram of the first stage of an 11-bit pipeline ADC, along with a BE-ADC of 10-bit linearity. The concept of the algorithm is explained considering a generalized block diagram of a switched capacitor pipeline stage. In latter sections, detailed analysis on two different types of pipeline stage topologies, viz. capacitor flip-over and capacitor nonflipover types will be discussed in detail. As gain of pipeline stage for both these topologies depend upon the ratio of the two constituent capacitors, they have been indicated as $C_{S}$ and $C_{F}$ in Fig. 2. The basic idea behind the calibration algorithm is to use a DC signal at the ADC input, which has a defined digital output. Corresponding BE-ADC output of that signal is $D_{B E}$. 
Due to the non-ideal nature of the first pipeline stage $D_{B E}$ is

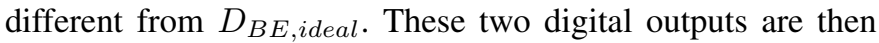
compared by a 10-bit digital comparator, which activates a control logic, associated with the stage capacitors $C_{S}$ and $C_{F}$. In [11], in order to achieve a pipeline stage gain of exactly 2 , amplifier gain was varied. In this paper, instead of controlling the amplifier gain, magnitude of the capacitor ratio, $C_{S} / C_{F}$ is controlled using a unit capacitor bank. However, in order to achieve that, a calculation of the desired amplifier gain is necessary. In the following sections, the two topologies of a switched-capacitor based MDAC circuit is discussed.

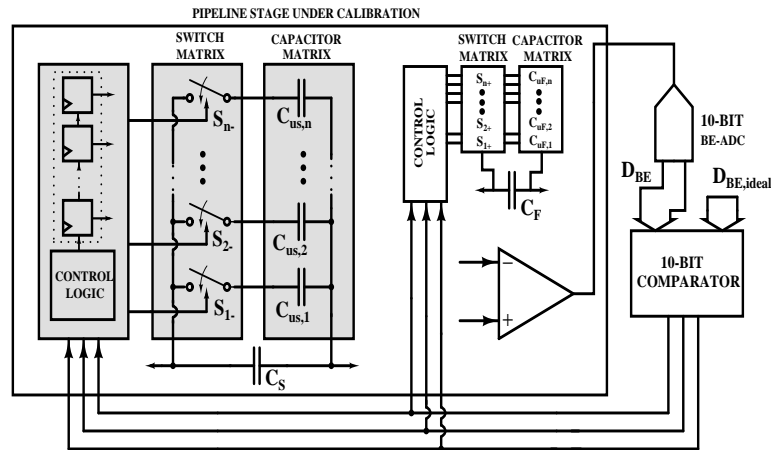

Fig. 2: Block Diagram Representation of Calibration Technique A. Capacitor Flip Over Topology:

A capacitor flip around pipeline stage shown in Fig. 3. The residue $\left(V_{\text {residue }}\right)$ signal expression can be written as [5],

$$
\begin{aligned}
V_{\text {residue }}= & \left(\frac{C_{S}+C_{F}}{C_{F}+\frac{1}{A}\left(C_{S}+C_{F}+C_{P}\right)}\right) V_{i n} \\
& -\left(\frac{C_{S}}{C_{F}+\frac{1}{A}\left(C_{S}+C_{F}+C_{P}\right)}\right) D_{i} \frac{V_{R}}{2}
\end{aligned}
$$

where $D_{i} \mathrm{~s}$ are the digital output of the $i_{t h}$ pipeline stage. $A$ is the amplifier gain, $C_{P}$ is the parasitic capacitence. $C_{S}$ $=(1+\gamma) C_{F}$, where $\gamma$ is the mismatch factor. Ideally the residue voltage expression for 1.5 bit stage can be written as, $V_{\text {residue }}=2 V_{i n}-V_{D A C}$, where $V_{D A C}$ is $D_{i} V_{R} / 2$. Equating coefficient of $V_{i n}$ to 2 and solving for $A$,

$$
A=2\left(\frac{C_{F}(2+\gamma)+C_{P}}{\gamma C_{F}}\right)
$$

Substituting " $A$ " into the co-efficient of $V_{D A C}$ and solving,

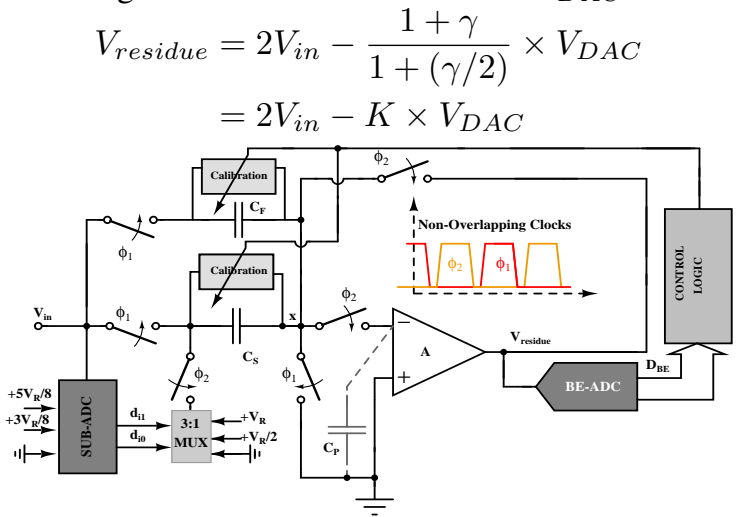

Fig. 3: First Stage MDAC Showing Flip Around Topology

Under ideal condition, $\gamma=0$ leads to $K_{\text {ideal }}=1$. However for $\gamma \neq 0$, the non-ideal sub-DAC output distorts the residue charecterictics. By replacing $\mathrm{K}$ in eqn. 3 with $K=K_{\text {ideal }}+$ $K_{D-E r r o r}$. In case of a flip-around MDAC topology, it is known that

$$
V_{D A C}= \begin{cases}0 & 0 \leq V_{\text {in }} \leq 3 V_{R} / 8 \\ V_{R} / 2 & 3 V_{R} / 8<V_{\text {in }} \leq 5 V_{R} / 8 \\ V_{R} & 5 V_{R} / 8<V_{\text {in }} \leq V_{R}\end{cases}
$$

For a input dynamic range of $V_{R}$, it is required to find out the linear and non-linear parts of the term $K_{D-E r r o r} \times V_{D A C}$ [16]. To achieve that $K_{D \text {-error }} \times V_{D A C}$ is segregated to,

$$
K_{D-E r r o r} V_{D A C}=\alpha_{1} V_{i n}+\beta_{1, V_{i n}}
$$

Plot of $K_{D-E r r o r} V_{D A C}$ is shown in Fig. 4(a), where $\alpha_{1} V_{i n}$ represents the linear term and $\beta_{1, V_{i n}}$ represents the non-linear function. To find $\alpha_{1}$, the mean absolute value of $\beta_{1, V_{i n}}$ is calculated from eqn. (4) for $V_{R}=1 V$,

$$
\begin{aligned}
\int_{0}^{V_{R}}\left|\left(\beta_{1, V_{i n}}\right)\right| d V_{\text {in }}= & \int_{0}^{V_{R}}\left|-\left(K_{D-\text { Error }}\right) V_{D A C}\right| d V_{\text {in }} \\
& +\int_{0}^{V_{R}}\left|-\alpha_{1} V_{\text {in }}\right| d V_{\text {in }} \\
= & 0.5 K_{D-\text { Error }}+0.5 \alpha_{1}
\end{aligned}
$$

Fig. 4: Flip Over MDAC Sub-DAC Error Charecterictics

The constant $\alpha_{1}$ in eqn. (5) is determined such that the mean absolute value of $\beta_{1, V_{i n}}$ is minimized [16]. From Eqn. (5), $\alpha_{1}$ is calculated as $\alpha_{1}=-K_{D-E r r o r}$ and $\beta_{1, V_{i n}=V_{R}}(=$ $\left.\beta_{1, V_{\text {in }}, \max }\right)$ can be calculated from eqn. (4) as,

$$
\begin{aligned}
\beta_{1, V_{\text {in }}, \max } & =\left(K_{D-\text { Error }} V_{R}\right)-\left(-K_{D=\text { Error }}\right) V_{R} \\
& =2 K_{D-\text { Error }} V_{R}
\end{aligned}
$$

Considering a linear BE-ADC, INL of the 11 bit ADC will be equal to $\left|\beta_{1, V_{i n}, \max }\right| / L S B$. For $V_{R}=1 V$, with an allowable ADC INL value of $1 L S B$, the maximum of $K_{D-E r r o r}$ value is found out from eqn. (6) as,

$$
\begin{aligned}
1 & =\frac{2\left(K_{D-\text { Error }} V_{R}\right)}{1 L S B} \\
& \Longrightarrow K_{D-\text { Error }, \text { max }}=0.0003
\end{aligned}
$$

Eqn. (7) gives the maximum allowable value of $K$ as 1.0003. Using Eqn. (1) and (2) Table II is formed, which gives the choice of $A$ and $C_{S} / C_{F}$.

TABLE II: Identification of the optimum $\left(C_{S} / C_{F}\right)$ and $A$ value

\begin{tabular}{|c|c|c|c|c|c|}
\hline$\frac{C_{S}}{C_{F}}$ & $C_{P}$ & $A(\mathrm{~V} / \mathrm{V})$ & $V_{\text {in }}$ Co-eff. & $\left|K_{D-\text { Error }}\right|$ & $\begin{array}{c}\text { ADC } \\
\text { SNDR }\end{array}$ \\
\hline 1.0002 & $10 \mathrm{fF}$ & 490 & 1.9918 & 1.0001 & $60.51 \mathrm{~dB}$ \\
\hline 1.0006 & $10 \mathrm{fF}$ & 490 & 1.9925 & 1.0003 & $61.35 \mathrm{~dB}$ \\
\hline 1.0006 & $10 \mathrm{fF}$ & 550 & 1.9935 & 1.0003 & $62.07 \mathrm{~dB}$ \\
\hline
\end{tabular}

B. Capacitor Non-Flip Over Topology:

A practical capacitor non-flip around pipeline stage shown in Fig. 5. Using the component parameters, the residue expression can be written as

$$
V_{\text {residue }}=\frac{C_{S}}{C_{F}+\frac{1}{A}\left(C_{S}+C_{F}+C_{P}\right)} \times\left(V_{\text {in }}-D_{i} \frac{V_{R}}{4}\right)
$$

Considering $V_{i n}$ as the input of a 1.5 bit non-flip around stage of MDAC gain $G$, the output signal can be written as, 


$$
\begin{aligned}
V_{\text {residue }} & =G\left(V_{\text {in }}-V_{D A C}\right) \\
& =\left(K_{G-\text { ideal }}+K_{G-\text { Error }}\right)\left(V_{\text {in }}-V_{D A C}\right)
\end{aligned}
$$

For non-ideal cases, $G$ deviates from ideal value of ' 2 ' due to non-zero $K_{G-E r r o r}$ value and distorts the ADC output. From [16], the term $K_{G-E r r o r} \times V_{D A C}$ in the eqn. (9) determines the non-linearity error of the stage. For a non-flip around stage with dynamic range $V_{R}, V_{D A C}$ is defined as,

$$
V_{D A C}= \begin{cases}0 & 0 \leq V_{i n} \leq 3 V_{R} / 8 \\ V_{R} / 4 & 3 V_{R} / 8<V_{i n} \leq 5 V_{R} / 8 \\ V_{R} / 2 & 5 V_{R} / 8<V_{i n} \leq V_{R}\end{cases}
$$

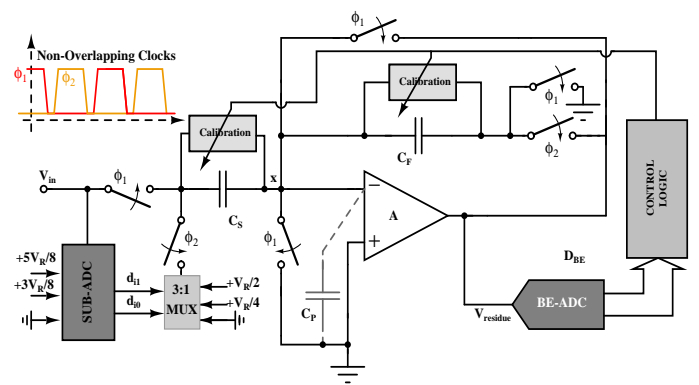

Fig. 5: First Stage MDAC Showing Non-Flip Around Topology

Hence, to evaluate the effect of $K_{G-E r r o r}$ on the ADC output linearity, ADC INL will be estimated in terms of $K_{G-\text { Error. }}$ As shown in eqn. (4), $K_{G-\text { error }} \times V_{D A C}$ can be seperated to,

$$
K_{G-E \text { Eror }} V_{D A C}=\alpha_{2} V_{i n}+\beta_{2, V_{i n}}
$$

To determine $\alpha_{2}$, the mean absolute value of $\beta_{2, V_{i n}}$ is calculated from eqn. (10) With $V_{R}=1 \mathrm{~V}$,

$$
\int_{0}^{V_{R}}\left|\left(\beta_{2, V_{i n}}\right)\right| d V_{i n}=0.25 K_{G-\text { Error }}+0.5 \alpha_{2}
$$

From Eqn. (11), $\alpha_{2}$ is calculated as $\alpha_{2}=-0.5 K_{G-E r r o r}$ [16]. $\beta_{2, V_{i n}}=V_{R}\left(=\beta_{2, V_{i n}, \max }\right)$ can be calculated from eqn. (10) as,

$$
\beta_{2, V_{\text {in }}, \max }=1.5 K_{G-E r r o r} V_{R}
$$

The ADC INL in terms of $K_{G-E \text { rror }}$ is estimated as $\left(1.5 \times K_{\text {Gain-Error }} V_{R}\right) / L S B$. Considering ADC INL of $1 L S B$, maximum $K_{G-E r r o r}$ value is found out as,

$$
\begin{aligned}
1 & =\frac{1.5\left(K_{G-\text { Error }} V_{R}\right)}{1 L S B} \\
& \Longrightarrow K_{G-\text { Error } \text { max }}=0.0004
\end{aligned}
$$

If, $G=K_{G-\text { ideal }}=2$ is set, from eqn.(13), theoritically INL of the 11 bit ADC becomes $0 L S B$. Taking $C_{P}=10 \mathrm{fF}$, estimated values of $C_{s} / C_{f}$ and amplifier gain of a front-end non-flip-around MDAC are tabulated in Table III.

TABLE III: Identification of the optimum $\left(C_{S} / C_{F}\right)$ and $A$ value

\begin{tabular}{|c|c|c|c|c|}
\hline$\frac{C_{S}}{C_{F}}$ & $A(\mathrm{~V} / \mathrm{V})$ & $V_{\text {in }}$ Co-eff. & $\left|K_{G-\text { Error }}\right|$ & ADC SNDR (dB) \\
\hline 2.26 & 24.95 & 1.9996 & 0.0004 & 67.54 \\
\hline $\mathbf{2 . 2 6}$ & $\mathbf{2 5}$ & $\mathbf{2 . 0 0 0 0}$ & $\mathbf{0 . 0 0 0 0}$ & 67.99 \\
\hline 2.26 & 25.04 & 2.0004 & 0.0004 & 67.81 \\
\hline
\end{tabular}

Comparing Table II and Table III, it is evident that in order to achieve 11 bit linearity of the ADC, non-flip-around topology provides a more attractive proposition, which requires a very low value of amplifier gain. This advantage provides the designer a flexibility in power-speed trade-off. These tables further show that in order to achieve an INL $<1 L S B$, nonflipover MDAC topology achieves better SNDR value than flip-over topologies at a much lesser amplifier gain value.

\section{Adaptive MDAC Gain Logic Controller Mechanism}

Fig. 6 shows the MDAC gain tuner logic controller. In general for an $N$ bit pipeline ADC, a $(N-i)$ bit BE-ADC response is utilized to calibrate the $i_{t h}$ stage [8]. In this diagram, a 1.5 bit front end pipeline stage of a 11 bit pipeline $\mathrm{ADC}$ has been calibrated using a 10-bit BE-ADC. In order to provide a low power solution of ADC non-linearity error calibration, a non-flip-around topology of the MDAC has been chosen. For such type of MDAC, from Table III, it is observed that when $C_{S} / C_{F}=2.26$, pipeline stage amplifier gain must be at $A=25 \mathrm{~V} / \mathrm{V}$ to obtain an MDAC gain of $2 \mathrm{~V} / \mathrm{V}$.

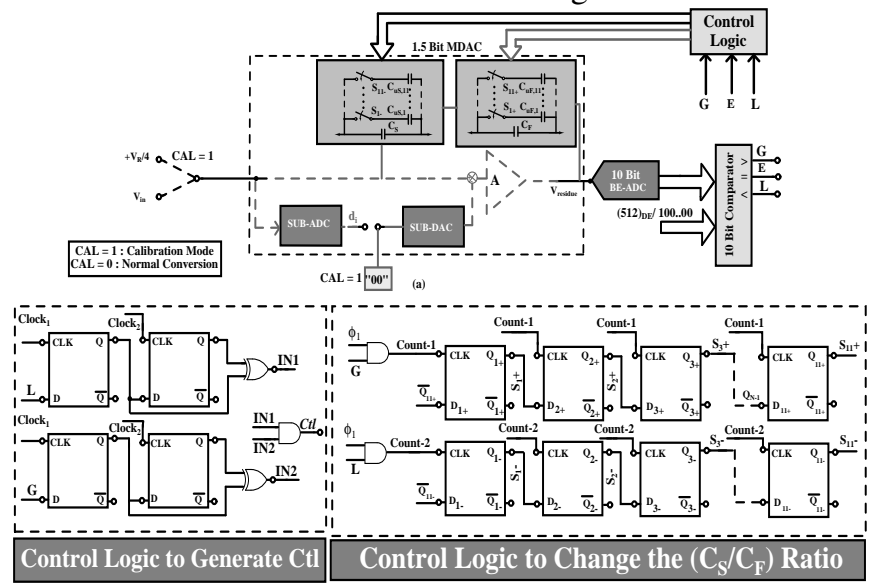

Fig. 6: MDAC Gain Controller Block Diagram

In practical circuits, it is difficult to maintain an open-loop amplifier gain which remains consistent with Process, Voltage and Temperature (PVT) variations. Hence, the proposed feedback mechanism ensures an MDAC gain of $2 \mathrm{~V} / \mathrm{V}$, by adaptively tuning the MDAC voltage gain of the pipeline stage by changing the desired capacitor ratio. To start the calibration, control signal $(C A L)$ goes high and a input voltage of $V_{R} / 4$ is applied at the input of the pipeline ADC. Sub-ADC (S-ADC) output of of the front-end pipeline stage is fed with a forced input of 00 (Fig. 6(a)). For this condition, the residue output of the front end pipeline stage can be written from eqn. (8) as,

$$
V_{\text {residue }}=\left(\frac{C_{S}}{C_{F}+\frac{1}{A}\left(C_{S}+C_{F}+C_{P}\right)}\right) \times \frac{V_{R}}{4}
$$

This residue signal, when fed to the $\mathrm{BE}-\mathrm{ADC}$, under non-ideal condition deviates from the ideal $D_{B E-A D C \text {,ideal }}$, $(512)_{D E}$. A 10- bit digital comparator is employed to detect this error by giving three possible decisions $(G, E, L)$ as outputs. Based on these signals, the digital logic block tunes the MDAC gain. As explained in Fig. 2, an additional $[n \times n]$ sized unit capacitor array $\left(C_{u S, 1} \ldots C_{u S, n}\right)$ and $\left(C_{u F, 1} \ldots C_{u F, n}\right)$ are used to gradually adjust the MDAC gain towards its ideal value of 2 to linearize the ADC output.

Fig. 6 (b) shows control logic to change the $C_{S} / C_{F}$ ratio. Two 11 bit johnson-ring counter is utilized to supply the control signals for two switch array $\left(S_{1}+\ldots . . S_{11}+\right)$ and $\left(S_{1}-\ldots . S_{11}-\right)$ as per the status of $G$ and $L$ signal. For example, considering an initial amplifier open loop gain $=20 \mathrm{~V} / \mathrm{V}$, the digital comparator gives " $L$ " as logic high as $D_{B E-A D C}$ $<(512)_{D E}$. Count $_{2}=\left(\phi_{1} . L\right)$ signal triggers the ring counter to start counting. Consequently, $V_{\text {residue }}$ gradually increases to $(512)_{D E}$ by adding 10 equally sized unit capacitors $\left(C_{u S, 1}\right.$ 
to $C_{u S, 10}$ ) to $C_{S}$ in 10 clock cycles. At $11_{t h}$ clock cycle, $V_{\text {residue }}$ becomes $(512)_{D E}+1 L S B$ and this in turn makes "G" logic high and " $L$ " logic low. This transition of $V_{\text {residue }}$ is detected by the control logic as shown in Fig. 6(c). To detect the transition of $V_{\text {residue }}$ signal using $C l o c k_{1}$ and $C l o c k_{2}$, two successive samples of $G$ and $L$ signals are fetched through DFFs, generating $I N 1$ and $I N 2$. Hence, a logic high pulse will be produced in $C t l$ signal whenever there is opposite logic of $G$ and $L$ signal in two consecutive samples. The status of switch control signals $\left[\left(S_{1}+\ldots . . S_{11}+\right)\right.$ and $\left.\left(S_{1}-\ldots . . S_{11}-\right)\right]$ at $V_{\text {residue }}=\left((512)_{D E}-1 L S B\right)$ get stored in two 11 bit register at the positive edge of $C t l$ signal, which is finally used to linearize the ADC ouput for signal input conversion. The corresponding timing diagram depicting the calibration mechanism is shown in Fig. 8.

\section{IMPLEMENTATION OF THE CIRCUIT}

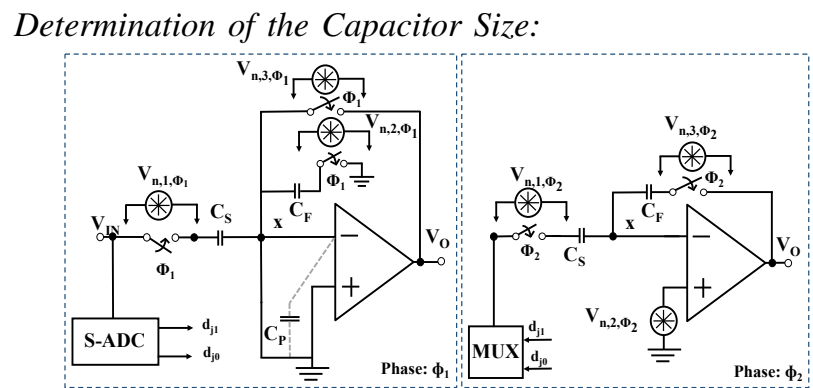

Fig. 7: Noise model of the front-end stage MDAC

Fig. 7 shows the noise model of the non flip around front end MDAC stage, which will be used to estimate the component values of $C_{S}$ and $C_{F}$. In the two phases the total input referred noise can be calculated as [5],

$$
V_{t o t, i n}^{2}=\left(\frac{C_{S} K T}{2 C_{F}^{2}}\right)+\frac{2}{3} \frac{K T\left(1+n_{t}\right)}{C_{S}+\beta_{2} C_{S}}\left(\frac{C_{S}+C_{F}}{C_{F}}\right)
$$

Taking, $C_{S}=2.26 C_{F}$ (Table III), $\beta_{2}$ can be calculated as 0.30. For single stage low power opamp, $n_{t}=2$ [5]. From eqn (15), for $C_{S}=2.26 C_{F}$ and $\beta_{2}=0.3, V_{\text {tot,in }}^{2}=\frac{3.36 K_{T}}{C_{F}}$. To minimize these noise at the ADC output, $V_{\text {tot, in }}^{2}<(0.5 \times$ Quantization noise) is considered. Hence for a 11 bit ADC having input dynamic range of $V_{R}=1 \mathrm{~V}$, the minimum value of $C_{F}$ and $C_{S}$ are calculated as, $C_{F, \min }=1.37 \mathrm{pF}$ and $C_{S, \min }=3.1 p F$ respectively.

For a 11 bit ADC with calibration input of $V_{R} / 4 \mathrm{~V}$, for $+1 L S B$ deviation of $V_{\text {residue }}$, from eqn. (14) the first stage MDAC gain becomes $\approx(.501 \mathrm{~V} / .250 \mathrm{~V})=2.004 \mathrm{~V} / \mathrm{V}$. Similarly for $-1 L S B$ deviation of $V_{\text {residue }}$, the MDAC gain value is found out as $\approx(.499 \mathrm{~V} / .250 \mathrm{~V})=1.996 \mathrm{~V} / \mathrm{V}$. Simulation has been done using eqn. (8) to estimate the $C_{S}, C_{F}$ value for $\pm 1 L S B$ change in $V_{\text {residue }}$ as shown in Table IV.

TABLE IV: Estimation of $C_{\mu S}$ and $C_{\mu F}$ values

\begin{tabular}{|c|c|c|c|c|}
\hline$C_{P}$ & $C_{S}$ & $C_{F}$ & $A$ & MDAC Gain \\
\hline $10 \mathrm{fF}$ & $3.1000 \mathrm{pF}$ & $1.3708 \mathrm{pF}$ & $25.5 \mathrm{~V} / \mathrm{V}$ & $\approx 2.004 \mathrm{~V} / \mathrm{V}$ \\
\hline $10 \mathrm{fF}$ & $3.1000 \mathrm{pF}$ & $\mathbf{1 . 3 7 4 8} \mathrm{pF}$ & $25.5 \mathrm{~V} / \mathrm{V}$ & $2 \mathrm{~V} / \mathrm{V}$ \\
\hline $10 \mathrm{fF}$ & $3.1000 \mathrm{pF}$ & $1.3708 \mathrm{pF}$ & $24.5 \mathrm{~V} / \mathrm{V}$ & $\approx 1.996 \mathrm{~V} / \mathrm{V}$ \\
\hline $10 \mathrm{fF}$ & $\mathbf{3 . 1 0 6 5} \mathrm{pF}$ & $1.3708 \mathrm{pF}$ & $24.5 \mathrm{~V} / \mathrm{V}$ & $2 \mathrm{~V} / \mathrm{V}$ \\
\hline
\end{tabular}

Hence from Table IV, the required unit capacitor value to be added in parallel with the existing $C_{F}$ to compensate the $0.5 \mathrm{~V} / \mathrm{V}$ change in amplifier open loop gain is found out as $4 f F\left(C_{u F}\right)$. Similarly from Table IV, the unit capacitor required to be added in parallel with the existing $C_{S}$ is determined as $6.5 f F\left(C_{u S}\right)$. In the implementation of the pipeline stage, $C_{\mu S}$ and $C_{\mu F}$ capacitor bank require 11 number of unit capacitors in each bank respectively. The switches of the capacitor bank have been implemented using typical transmission gate topology with NMOS $(\mathrm{W} / \mathrm{L})=2 / 0.18$, and PMOS $(\mathrm{W} / \mathrm{L})=2 / 0.18$ respectively. Hence the additional hardware, comprising of $C_{\mu S, \text { total }}=3.1715 \mathrm{pF}, C_{\mu F, \text { total }}=1.414 \mathrm{pF}$ and the switching network consumes a very nominal extra die area.

\section{Simulations RESUlts}

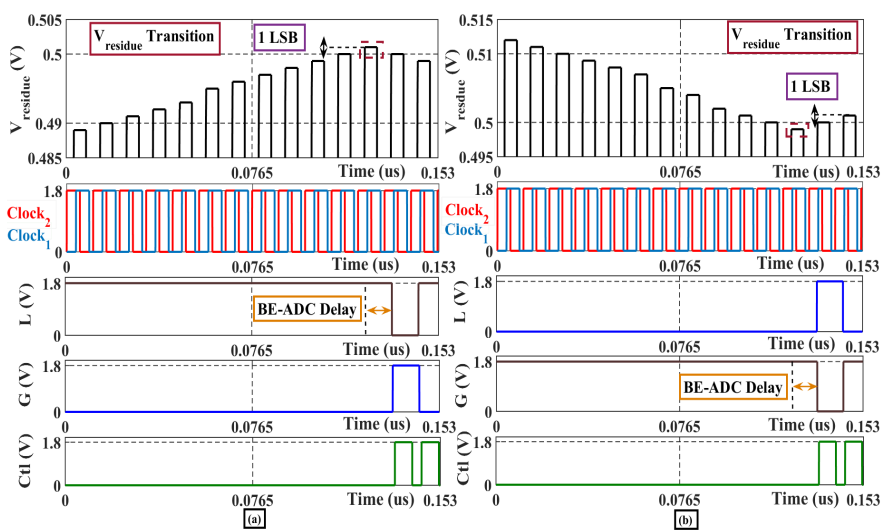

Fig. 8: Non Flip Around Topology Timing Diagram (a) for $A=$ $20 \mathrm{~V} / \mathrm{V}$ (b) for $A=30 \mathrm{~V} / \mathrm{V}$

Fig. 8(a,b) shows the transient plots of $V_{\text {residue }}, C l o c k_{1}$, Clock $_{2}, L, G$ and $c t l$ signal for initial amplifier gain $\left(A_{i n i}\right)$ of $20 \mathrm{~V} / \mathrm{V}$ and $30 \mathrm{~V} / \mathrm{V}$. For $\pm 20 \%$ variation of amplifier gain, in worst case $14 \mathrm{ADC}$ clock cycles are required for the calibration scheme to converge to the ideal MDAC gain (Fig. 8). Hence, negligible halt time of normal ADC conversion is required. Simulation results of a 11 bit pipeline ADC in an $180 \mathrm{~nm}$ CMOS process are presented. The first $1.5 \mathrm{bit}$ stage is treated with non ideal conditions (low amplifier gain, capacitor mis-match) and and rest of the stages are modelled with ideal conditions and resolve 10 bits of data. For an input signal dynamic range of $1 V_{P-P}$ and clock frequency of $100 \mathrm{MHz}$, Fig. 9 shows the the pre and post calibration SNDR plots of the 11 bit pipeline ADC output sampled at nyquist rate. Considering non-flip around MDAC topology, for an initial amplifier open loop gain $\left(A_{\text {ini }}\right)$ of $20 \mathrm{~V} / \mathrm{V}$ and $30 \mathrm{~V} / \mathrm{V}$, the SNDR value gets improved to $65.13 \mathrm{~dB}(10.52$ ENOB) from $46.21 \mathrm{~dB}$ (7.38 ENOB) (Fig. 9 (a)) and 65.16 $\mathrm{dB}$ (10.53 ENOB) from $44.23 \mathrm{~dB}$ (7.05 ENOB) (Fig. 9 (b)) post calibration respectively.

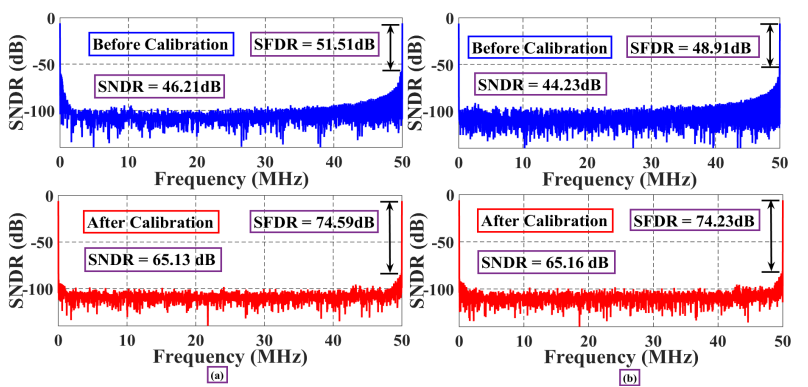

Fig. 9: Before and after calibration SNDR plots of the ADC (a) for $A=20 \mathrm{~V} / \mathrm{V}$ (b) for $A=30 \mathrm{~V} / \mathrm{V}$

Fig. 10 shows the before and after calibration diffrential non-linearity (DNL) and integral non-linearity (INL) plots of the 11-bit ADC under calibration. It is found that, with an 
initial amplifier $\mathrm{O} / \mathrm{L}$ gain value of $30 \mathrm{~V} / \mathrm{V}$, after calibration the INL of the ADC gets improved to $-1 L S B \leq I N L \leq$ $+1 L S B$ from $-24.2 L S B \leq I N L \leq 0 L S B$. Fig. 11 shows the plot of SNDR variation with respect to variation in $C_{S} / C_{F}$ ratio $( \pm 2.5 \%)$ and amplifier gain $( \pm 20 \%)$ before calibration (Fig. 11(a)) and after calibration (Fig. 11(b)). Plot shows that for all cases the post calibration SNDR of the 11 bit ADC in calibration achieves a minimum value of $64.11 \mathrm{~dB}$.

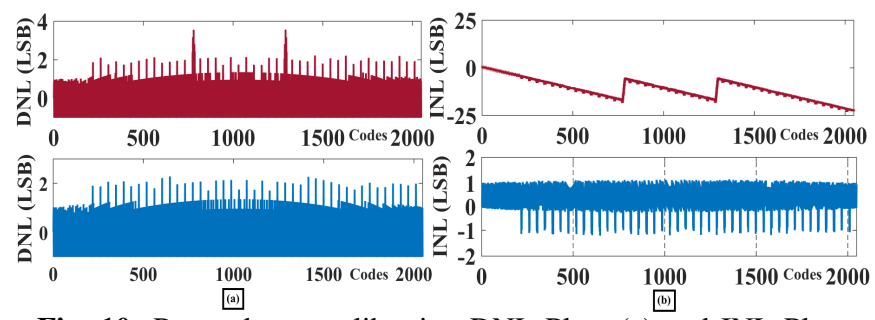

Fig. 10: Pre and post calibration DNL Plots (a) and INL Plots (b) of the ADC for $A=30 \mathrm{~V} / \mathrm{V}$

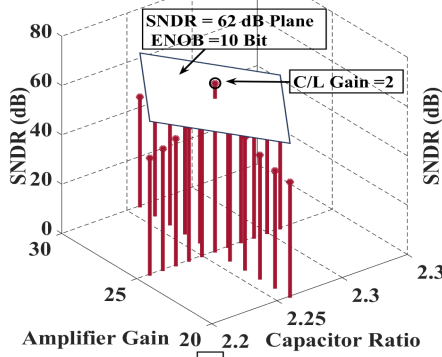

(a)

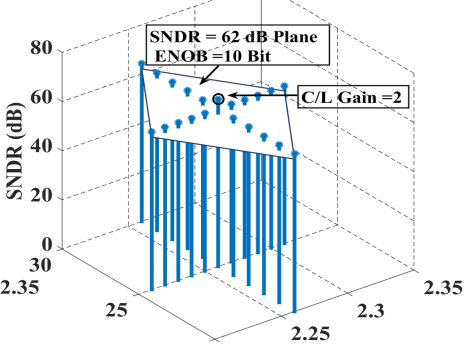

(b)
Fig. 11: SNDR variation w.r.t to $C_{S} / C_{F}$ ratio and amp. gain (a) before calibration (b) after calibration

A. Variation of Calibration I/P Due to Noise and Temp. Drift:

Table $\mathrm{V}$ shows the ADC SNDR under the influence of varying calibration signal input. However, the allowable range of the deviation of calibration signal can be increased further by adding additional unit capacitors in the unit capacitor array.

TABLE V: Cal. I/P Variation vs Post Cal. ADC SNDR \& INL

\begin{tabular}{|c|c|c|c|c|}
\hline Cal. Sig. Var. & ADC SNDR & ADC INL Max & $C_{u s, t o t}$ & $C_{u F, t o t}$ \\
\hline$\approx \pm 1 \% \mathrm{~V}$ & $65.43 \mathrm{~dB}$ & $\pm 1 \mathrm{LSB}$ & $3.19 \mathrm{pF}$ & $1.42 \mathrm{pF}$ \\
\hline$\approx \pm 2 \% \mathrm{~V}$ & $65.39 \mathrm{~dB}$ & $\pm 1 \mathrm{LSB}$ & $3.20 \mathrm{pF}$ & $1.43 \mathrm{pF}$ \\
\hline$\approx \pm 3 \% \mathrm{~V}$ & $65.38 \mathrm{~dB}$ & $\pm 1 \mathrm{LSB}$ & $3.21 \mathrm{pF}$ & $1.44 \mathrm{pF}$ \\
\hline$\approx \pm 5 \% \mathrm{~V}$ & $65.36 \mathrm{~dB}$ & $\pm 1 \mathrm{LSB}$ & $3.24 \mathrm{pF}$ & $1.46 \mathrm{pF}$ \\
\hline
\end{tabular}

\section{B. Variation of Unit Capacitor Ratio in Non-Ideal Conditions:}

The resiliency of the proposed algorithm in practical scenario is further checked against the random variation in $C_{\mu s} / C_{\mu f}$ from its ideal value of "1.625" (refer section III). Fig. 12 shows the plot of ADC SNDR with respect to gaussian distributed randomly varying $C_{u s} / C_{u f}$ ratio. Plot shows the efficacy of the calibration algorithm for $+/-68 \%$ variation of $C_{\mu s} / C_{\mu f}$ ratio.

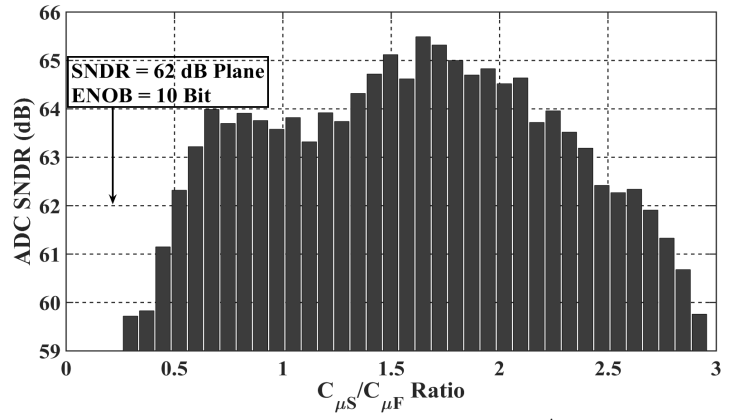

Fig. 12: SNDR variation w.r.t to $C_{u S} / C_{u F}$ ratio
C. Varaition of the ADC Clock Period (Random Clock Jitter)

Timing jitter analysis of the ADC has been done with input clock jitter of $2 \%, 5 \%, 10 \%$ variation at nyquist sampling rate (Table VI). Table VI shows that in all cases after calibration SNDR value denotes ENOB $>10$ Bit.

TABLE VI: ADC Input Clock Jitter \% vs ADC SNDR

\begin{tabular}{|c|c|}
\hline Clock Jitter (\%) & ADC SNDR \\
\hline $2.5 \%$ & $62.25 \mathrm{~dB}$ \\
\hline $5 \%$ & $62.23 \mathrm{~dB}$ \\
\hline $10 \%$ & $62.14 \mathrm{~dB}$ \\
\hline
\end{tabular}

\section{CONCLUSION}

In this paper, a low power foreground calibration technique of a pipeline ADC has been developed. The proposed algorithm employs a feedback mechanism to dynamically adjust the MDAC gain towards the ideal value to calibrate the ADC output. An 11-bit pipeline ADC has been simulated in $0.18 \mu \mathrm{m}$ CMOS process technology. The post calibration simulation results of the 11 bit ADC prooves that the proposed technique is capable of providing requisite SNDR considering variation in the values of amplifier gain $(A)$ and capacitor ratio $\left(C_{S} / C_{F}\right)$.

\section{REFERENCES}

[1] Maliang Liu , Dengquan Li , and Zhangming Zhu, "A Dual-Supply TwoStage CMOS Op-amp for High-Speed Pipeline ADCs Application”, IEEE TCAS-II: Express Briefs, VOL. 67, NO. 4, pp. 650-654, APRIL 2020.

[2] T. Hung, F. Liao, and T. Kuo, "A 12-Bit Time-Interleaved 400-MS/s Pipelined ADC With Split-ADC Digital Background Calibration in 4,000 Conversions/Channel", IEEE TCAS-II: Express Briefs, vol. 66, no. 11, pp. 1810-1814, Nov. 2019.

[3] P. Yang, X. Wang, C. Wang, F. Li, H. Jiang and Z. Wang, "A 14-bit 200-Ms/s SHA-Less Pipelined ADC With Aperture Error Reduction," in IEEE TVLSI, vol. 28, no. 9, pp. 2004-2013, Sept. 2020.

[4] Imran Ahmed. 2010. Pipelined ADC Design and Enhancement Techniques (1st. ed.). Springer Publishing Company, Incorporated.

[5] Gustavsson, Mikael, Wikner, J. Jacob, Nianxiong Tan. (2002). CMOS Data Converters for Communications.

[6] Jipeng $\mathrm{Li}$ and Un-Ku Moon, "Background Calibration Techniques for Multistage Pipelined ADCs With Digital Redundancy", IEEE TCAS-II: Analog Signal Processing, vol. 50, no. 9, pp. 531-538, Sep. 2003.

[7] John P. Keane, Paul J. Hurst, Stephen H. Lewis, "Background Interstage Gain CalibrationTechnique for Pipelined ADCs", IEEE TCAS-I: Regular Papers, VOL. 52, NO. 1, pp. 32-43, JANUARY 2005.

[8] Dong-Young Chang, J. Li, Un-Ku Moon, Radix-based digital calibration techniques for multi-stage recycling pipelined ADCs, IEEE TCAS I: Regular Papers, vol. 51, no. 11, pp. 2133-2140, Nov. 2004.

[9] A. Verma and B. Razavi, " A 10-Bit 500-MS/s 55-mW CMOS ADC," IEEE JSSC, VOL. 44, NO. 11, NOVEMBER 2009, pp. 3039-3050.

[10] Bibhu Datta Sahoo and Behzad Razavi,, A 12-Bit 200-MHz CMOS ADC, IEEE JSSC, VOL. 44, NO. 9, pp. 2366-2380, Sep. 2009.

[11] S. Roy and S. Chatterjee, "A Low Power mixed Signal Foreground Calibration Technique of a Pipeline ADC using a Variable Gain Amplifier," TENCON 2019, Kochi, India, 2019, pp. 2168-2172.

[12] S. Roy and S. Chatterjee, "Sinusoid Based Foreground Calibration Algorithm of a Pipeline ADC Using Time Averaged Radix Extraction," IEEE TENCON 2018, Jeju, Korea (South), pp. 1332-1337.

[13] S. Roy, B. Sahoo and S. Banerjee, "Radix based digital calibration technique for pipelined ADC using Nyquist sampling of sinusoid", IEEE ISCAS, Seoul, 2012, pp. 2985-2988.

[14] S. Chatterjee and S. Roy, "A Square Wave-Based Digital Foreground Calibration Algorithm of a Pipeline ADC Using Approximate Harmonic Sampling," in IEEE Transactions on Circuits and Systems II: Express Briefs, vol. 68, no. 4, pp. 1068-1072, April 2021.

[15] S. Roy and H. Basak, S. Banerjee, "Foreground calibration technique of a pipeline ADC using capacitor ratio of Multiplying Digital-to-Analog Converter (MDAC)," in Microelectronics Journal, Vol. 44, Issue 12, Dec. 2013, pp. 1336-1347.

[16] G. Nikandish, B. Sedighi and M. S. Bakhtiar, "INL Prediction Method in Pipeline ADCs," APCCAS 2006 - 2006 IEEE Asia Pacific Conference on Circuits and Systems, 2006, pp. 13-16. 